\title{
LATTICE ISOMORPHISMS OF FINITE NON-ABELIAN GROUPS OF EXPONENT $p$
}

\author{
R. F. SPRING
}

In this paper we give some lattice characterizations of finite nonabelian groups of exponent $p$ and investigate the extent to which a group of this type is determined by its lattice of subgroups. We also give a complete classification of all class 2 groups of exponent $p$ determined by four independent generators. This completes a partial classification of these groups given by Brahana [2]. In $\$ 3$ we prove that any class 2 group of exponent $p$ generated by fewer than five independent generators is completely determined by its lattice of subgroups.

We shall use $L(G)$ to denote the lattice of subgroups of a group $G$, and $\phi(G)$ to denote the Frattini subgroup ( $=\bigcap$ maximal subgroups of $G$ ). Two groups, $G$ and $H$, are said to be lattice-isomorphic, or $L$-isomorphic, if $L(G)$ is isomorphic to $L(H)$. A lattice $L$ is called irreducible if $L$ is not decomposable into a direct product of two or more lattices of dimension greater than one. $L$ is said to be a projective geometry if it is irreducible, complemented, and modular.

1. Lattice characterizations. It is a well-known fact that the lattice of subgroups of an elementary abelian group of order $p^{n}$ is an $(n-1)$ dimensional finite projective geometry over $G F(p)$. A finite group $G$ is called a $P$-group if it is either

(1) an elementary abelian group, or

(2) a group generated by $a_{1}, a_{2}, \cdots, a_{n}$, and $b$ with $a_{i}^{p}=b^{q}=1$, $a_{i} a_{j}=a_{j} a_{i}$, and $b a_{i} b^{-1}=a_{i}^{r}$ where $r^{q} \equiv 1(\bmod p), r \not \equiv 1(\bmod p)$, and $p$ and $q$ are primes such that $p \equiv 1(\bmod q)$.

If $G$ is an elementary abelian group of order $p^{n}$ and $H$ is a nonabelian $P$-group of order $p^{n-1} q$, then $G$ and $H$ are $L$-isomorphic. Baer has proven that the non-abelian $P$-groups are the only groups which are $L$-isomorphic to elementary abelian groups [1, Theorem 11.2]. Iwasawa [4] has shown further that $P$-groups are the only groups whose lattices of subgroups are irreducible, complemented, modular lattices. We summarize these facts in the following lemma.

Lemma 1. If $G$ is a finite group, then the following statements are equivalent:

Presented to the Society, January 23, 1961; received by the editors December 29, 1961. 
(i) $G$ is $L$-isomorphic to an elementary abelian group.

(ii) $G$ is a $P$-group.

(iii) $L(G)$ is a finite projective geometry over $G F(p)$.

The following defining relations give a generalization of the nonabelian $P$-group. Let $G$ be a finite group with a normal subgroup $N$ satisfying the following:

(1) $N$ is abelian, of exponent $p^{m}$ where $p$ is an odd prime.

(2) $G / N$ is cyclic, of exponent $q^{n}$ where $q$ is prime and $p \equiv 1(\bmod q)$.

(3) For any element $a$ in $N$, and any element $g$ of $G$ such that $g$ is not in $N, \mathrm{gag}^{-1}=a^{r}$ where $r$ is independent of $a$ and is a positive integer such that $r^{q} \equiv 1(\bmod p)$ and $r \neq 1$.

In the terminology of Suzuki, a group of the above type is called a $P^{*}$-group; a group in which $G / N$ is of exponent $q$ is called a $P_{1}^{*}$ group; a group in which $N$ is of exponent $p$ is called a $P_{0}^{*}$-group. A non-abelian $P$-group is both a $P_{1}^{*}$-group and a $P_{0}^{*}$-group.

A lattice shall be called chainfree if every ideal of dimension 2 is not a chain. Clearly, a chainfree lattice contains no ideals which are chains other than the trivial two element chains. $L(G)$ is said to be lower semi-modular if the intersection of two subgroups $A$ and $B$ is maximal in $B$ whenever $A$ is a maximal subgroup of $A \cup B$.

THEOREM 1. If $G$ is a finite group, then $G$ is non-abelian of exponent $p$ if and only if $L(G)$ is a nonmodular, chainfree, lower semi-modular lattice in which every interval is irreducible.

Proof. If $L(G)$ is chainfree, $G$ cannot have a cyclic subgroup of order $p^{2}$ and hence cannot have elements of order $p^{2}$. Suzuki [6, Proposition 1.5] has shown that when $L(G)$ is lower semi-modular with every interval irreducible and $G$ is of finite order, then $G$ is either a $p$-group or a $P_{1}^{*}$-group. Now if $G$ cannot have elements of order $p^{2}$, then $G$ must either be a $p$-group of exponent $p$ or a nonabelian $P$-group. Since $L(G)$ is nonmodular, $G$ cannot be abelian, nor can $G$ be a $P$-group. Therefore $G$ is a non-abelian group of exponent $p$.

Conversely if $G$ is non-abelian of exponent $p$, then $G$ has no cyclic subgroup of order $p^{2}$, and so $L(G)$ is chainfree. Since $G$ is a $p$-group, $L(G)$ is lower semi-modular with every interval irreducible. Due to a theorem by Iwasawa [4] the nature of all groups which have a modular lattice of subgroups is well known (see also [5, Theorem 3.3]). Any modular $p$-group which is of exponent $p$ is abelian. Hence $L(G)$ must be nonmodular. 
The following lemma gives two different lattice characterizations of a normal subgroup of any finite group of exponent $p$.

LEMMA 2. If $G$ is a finite group of exponent $p$ and $[M, N]$ is an interval of dimension $k$ in $L(G)$, then the following statements are equivalent:

(i) $N$ is a normal subgroup of $M$.

(ii) $[M, N]$ contains exactly $\left(p^{k}-1\right) /(p-1)$ atoms.

(iii) $M$ is the union of all the atoms of $[M, N]$.

Proof. (i) clearly implies (ii). Suppose (ii) is true. Let $T$ be the union of all the atoms of $[M, N]$. Since $G$ is a $p$-group and $N$ is maximal in every atom of $[M, N]$, then $N$ is normal in all of these atoms and therefore must be a normal subgroup of $T$. Then $T / N$ is a group of exponent $p$ and order $p^{k}$. If $N$ is of order $p^{a}$, then $T$ will be of order $p^{a+k}$. But since $[M, N]$ is of dimension $k$, then $M$ is also of order $p^{a+k}$. Since $T$ must be an element of $[M, N]$ then $T=M$. Therefore (ii) implies (iii). (iii) clearly implies (i) since $N$ is normal in every atom. Hence (i), (ii), and (iii) are equivalent.

The following lemma gives a lattice characterization of the abelian subgroups and factor groups of a group of exponent $p$, and also characterizes the center and commutator subgroup by lattice properties.

LEMMA 3. If $S$ is a subgroup of any finite group of exponent $p$ and $R$ is a subgroup of $S$ then

(1) $R$ is normal in $S$ and $S / R$ is abelian if and only if the lattice interval $[S, R]$ is a finite projective geometry over $G F(p)$.

(2) $R$ is a subgroup of the center of $S$ if and only if $L(R \cup A)$ is a finite projective geometry over $G F(p)$ for every atom $A$ of $L(S)$.

(3) If $S$ is non-abelian, $R$ is the commutator subgroup of $S$ if and only if $R=\bigcap$ maximal subgroups of $S$.

Proof. Suppose the index of $R$ in $S$ is $p^{n}$. If $[S, R]$ is a finite projective geometry over $G F(p)$, then $[S, R]$ contains $\left(p^{n}-1\right) /(p-1)$ atoms, and so by Lemma $2, R$ is normal in $S$. Furthermore, $L(S / R)$ $=[S, R]$ is modular. Therefore $S / R$ is abelian, since any modular group of exponent $p$ is abelian. This proves the sufficiency of the conditions in (1). The necessity of the conditions in (1) and the proof of (2) are obvious and follow directly from Lemma 1. To prove (3) we may observe that the intersection of all the maximal subgroups of $S$ is $\phi(S)$. Denote the commutator subgroup of $S$ by $K(S) . S / \phi(S)$ is abelian, hence $K(S)<\phi(S) . S / K(S)$ is also abelian, and so $[S, K(S)]$ and $[S, \phi(S)]$ are finite projective geometries over $G F(p)$. Now if 
$K(S) \neq \phi(S)$ then $[S, K(S)]$ would be a geometry of larger dimension than $[S, \phi(S)]$, and hence $S / K(S)$ would have more maximal subgroups than $S / \phi(S)$. But this is impossible since $\phi(S)$ is contained in every maximal subgroup of $S$. Therefore $K(S)=\phi(S)$.

2. Properties of $L$-isomorphisms. The results of the preceding section may be used to determine the nature of the $L$-isomorphisms of non-abelian groups of exponent $p$.

TheOREM 2. If $G$ is a finite non-abelian group of exponent $p$ and $\alpha$ is a lattice-isomorphism of $G$ onto a group $H$, then $H$ is also a finite non-abelian group of exponent $p$, and for any two subgroups $R$ and $S$ of $G$ :

(1) If $R<S$ then $[S: R]=[\alpha(S): \alpha(R)]$.

(2) $R$ is a normal subgroup of $S$ if and only if $\alpha(R)$ is a normal subgroup of $\alpha(S)$.

(3) $R$ is the center of $S$ if and only if $\alpha(R)$ is the center of $\alpha(S)$.

(4) $R$ is the commutator subgroup of $S$ if and only if $\alpha(R)$ is the commutator subgroup of $\alpha(S)$.

Proof. If $G$ is a finite non-abelian group of exponent $p$, then $H$ must be a finite non-abelian group of prime exponent since the lattice properties by which $G$ is characterized in Theorem 1 are invariant under any $L$-isomorphism. Furthermore, $G$ and $H$ must be of the same exponent $p$ since every subgroup of order $p^{2}$ in a group of exponent $p$ contains exactly $p+1$ subgroups of order $p$. Hence $G$ and $H$ are of the same order; and so clearly $\alpha$ must preserve the index of $R$ in $S$ whenever $R<S$. This proves (1). (2), (3), and (4) follow directly from Lemma 2 and (2) and (3) of Lemma 3 since the lattice properties by which these subgroups are characterized remain invariant under any $L$-isomorphism.

We shall now determine some conditions for a non-abelian group of exponent $p$ to be uniquely determined by its lattice of subgroups. This necessitates a closer examination of these groups. Much can be learned by studying the class 2 finite groups of exponent $p$ since this type of group is a factor group in every finite non-abelian group of exponent $p$.

3. Class 2 groups. In a class 2 finite group of exponent $p$, the commutator subgroup is equal to the Frattini subgroup and is contained in the center. A group of class 2 and exponent $p$ generated freely by $d$ independent generators, $u_{1}, u_{2}, \cdots, u_{d}$, is uniquely determined and is the group of maximum order with $d$ generators. This group is called the master group, and is denoted by $\$$. Since $d$ generators yield 
$d(d-1) / 2$ commutators of pairs of the generators, the order of $(5)$ is $p^{\phi+d}$ where $\phi=d(d-1) / 2$ and $p^{\phi}$ is the order of $\phi(\$)$. Any other class 2 group of exponent $p$ with $d$ independent generators can be specified by all the defining relations of $(5)$ and some additional relations in which one or more elements of the commutator subgroup are set equal to the identity. Hence if $G$ is any group of class 2 and exponent $p$ with $d$ independent generators, then $G$ is a factor group of $\$ 5$ modulo a suitable subgroup of $\phi(\$)$. The number of distinct groups possible is dependent on $d$ and the relations between the commutators and noncommutators in $\phi(\$)$.

In the cases of $d=2$ and $d=3$, every element of $\phi(\$)$ is a commutator. When $d=2$, $\&$ is a well-known group of order $p^{3}$, and this is the only class 2 group of exponent $p$ with 2 generators. When $d=3$, \& is of order $p^{6}$, and only two other groups are possible, one of order $p^{5}$ and one of order $p^{4}$. However when $d=4, B$ is of order $p^{10}$, and not every element of the commutator subgroup is a commutator. By first considering the possible ways in which the four generators can permute or not permute with each other, and then from these groups determining the distinct factor groups modulo subgroups of $\phi(G)$, the defining relations for all groups with $d=4$ can be found. We have used this method to determine all such groups. Our list verifies the correctness of Brahana's [2] classification; however, Brahana's classification is not complete. In simplifying the problem of classification, Brahana limited his study to those groups in which $\phi(G)=$ the center of $G$; for when $\phi(G)$ is properly contained in the center of $G$, then $G$ is a direct product of an elementary abelian group and a group $G^{\prime}$ with $d<4$ such that $\phi\left(G^{\prime}\right)=$ the center of $G^{\prime}$. He incorrectly assumed that in any class 2 group $G$ with $d=4$ of order $p^{k}$, the group $\phi(G)$ is properly contained in the center of $G$ when $k<7$. Hence his classification includes all the groups of orders $p^{10}, p^{9}, p^{8}$, and $p^{7}$; but he stopped at this point and hence did not discover the groups of orders $p^{6}$ and $p^{5}$ in which $\phi(G)=$ the center of $G$. These new groups are numbered 14., 15., 16., and 18. in our list and complete the partial classification given by Brahana [2, p. 376].

For any odd prime $p$, there are nineteen class 2 groups of exponent $p$ with four independent generators. If the master group is specified by

$$
\begin{aligned}
& \text { (S) }=\left\{u_{1}, u_{2}, u_{3}, u_{4}\right\} \quad s_{1}=u_{1}^{-1} u_{2}^{-1} u_{1} u_{2} \quad s_{4}=u_{2}^{-1} u_{3}^{-1} u_{2} u_{3} \\
& \phi(ङ)=\left\{s_{1}, s_{2}, s_{3}, s_{4}, s_{5}, s_{6}\right\} \quad s_{2}=u_{1}^{-1} u_{3}^{-1} u_{1} u_{3} \quad s_{5}=u_{2}^{-1} u_{4}^{-1} u_{2} u_{4} \\
& u_{i}^{p}=1 \text { and } u_{i} s_{j}=s_{j} u_{i} \quad s_{3}=u_{1}^{-1} u_{4}^{-1} u_{1} u_{4} \quad s_{6}=u_{3}^{-1} u_{4}^{-1} u_{3} u_{4}
\end{aligned}
$$

then the defining relations of all the groups with $d=4$ are as follows: 
order of $G$

$\begin{aligned} \text { 1. } & p^{10} \\ \text { 2. } & p^{9} \\ \text { 3. } & p^{9} \\ \text { 4. } & p^{8} \\ \text { 5. } & p^{8} \\ \text { 6. } & p^{8} \\ \text { 7. } & p^{8} \\ \text { 8. } & p^{7} \\ \text { 9. } & p^{7} \\ \text { 10. } & p^{7} \\ \text { 11. } & p^{7} \\ \text { 12. } & p^{7} \\ \text { 13. } & p^{7} \\ \text { 14. } & p^{6} \\ \text { 15. } & p^{6} \\ \text { 16. } & p^{6} \\ \text { 17. } & p^{6} \\ \text { 18. } & p^{5} \\ \text { 19. } & p^{6} \\ \text { 20. } & p^{4}\end{aligned}$

defining relations

$1=1$ (master group)

$s_{1}=1$

$s_{1} s_{6}=1$

$s_{1}=s_{2}=1$

$s_{1}=s_{6}=1$

$s_{1} s_{6}=s_{2}=1$

$s_{1} s_{6}=s_{2} s_{5}^{r}=1$ ( $r$ is nonsquare)

$s_{1}=s_{2}=s_{3}=1$

$s_{1}=s_{2}=s_{4}=1$

$s_{1}=s_{2}=s_{6}=1$

$s_{1} s_{6}=s_{2}=s_{3}=1$

$s_{1} s_{6}=s_{2}=s_{5}=1$

$s_{1} s_{6}=s_{2} s_{5}^{r}=s_{3}=1 \quad(r$ is nonsquare)

$s_{1} s_{6}=s_{2} s_{5}^{r}=s_{3}=s_{4}=1 \quad(r$ is nonsquare)

$s_{1} s_{6}=s_{2}=s_{3}=s_{5}=1$

$s_{1}=s_{2}=s_{5}=s_{6}=1$

$s_{1}=s_{2}=s_{3}=s_{6}=1$

$s_{1} s_{6}=s_{2}=s_{3}=s_{4}=s_{5}=1$

$s_{1}=s_{2}=s_{3}=s_{4}=s_{6}=1$

$s_{1}=s_{2}=s_{3}=s_{4}=s_{5}=s_{6}=1$ (abelian group) abelian subgroups, $A$ with $[A: \phi(G)]=p^{2}$

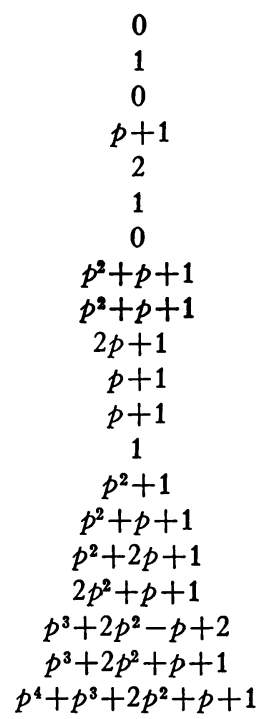

We have included in our classification those groups in which $\phi(G)$ is properly contained in the center (groups $8 ., 17$., and 19.), and also the elementary abelian group. By including these groups an interesting duality can be observed in the defining relations of our list. The group numbered $n$ corresponds to the group numbered $21-n$ when $n<8$, and the groups of order $p^{7}$ are self-dual. This duality serves to check the completeness of the list, and is moreover a duality of the spaces of Brahana's geometric description of the classification [2, p. 379].

Groups 11. and 12. differ in that group 17. is a factor group of 11., but is not a factor group of 12 . Groups 8 . and 9. have different indices of $\phi(G)$ in the center of $G$. The number of abelian subgroups in which $\phi(G)$ is of index $p^{2}$ serves to distinguish the remaining groups. For all of these groups, $L(G)$ is an interval [\$S, $S]$ in $L(\mathbb{B})$ for some suitable subgroup $S$ of $\phi(ङ)$. From these considerations and the very simple nature of the groups when $d=2$ and $d=3$, the following theorem follows as a consequence of Theorem 1 and Theorem 2.

THEOREM 3. If $G$ is a class 2 group of exponent $p$ with fewer than five independent generators and $H$ is a finite group, then $L(G)$ and $L(H)$ are isomorphic if and only if $G$ and $H$ are isomorphic.

When $d>4, L(G)$ may not determine $G$. In a recent paper [3], Brahana has classified a number of class 2 groups of exponent $p$ 
with five generators $u_{1}, \cdots, u_{5}$. All of the groups can be expressed in the following form when $p=11: s_{1} s_{8}=s_{2} s_{7}=s_{3} s_{9}^{\alpha}=s_{4} s_{6}^{5}=s_{5} s_{10}=1$ where $s_{i}=u_{\jmath}^{-1} u_{k}^{-1} u_{j} u_{k}, j<k, k=2,3,4,5$. Yff [7] has shown that the group defined by $\alpha=2$ in the above relations is not isomorphic to the group described when $\alpha=-2$, and yet the relations among the subgroups of these two groups are exactly identical.

\section{REFERENCES}

1. R. Baer, The significance of the system of subgroups for the structure of the group, Amer. J. Math. 61 (1939), 1-44.

2. H. R. Brahana, Finite metabelian groups and plucker line-coordinates, Amer. J. Math. 62 (1940), 365-379.

3. - On metabelian groups of order $p^{10}$ with five generators, Rend. Circ. Mat. Palermo 9 (1960), 1-17.

4. K. Iwasawa, Über die endlichen Gruppen und die Verbände ihrer Untergruppen, J. Fac. Sci. Imp. Univ. Tokyo Sect. I 4 (1941), 171-199.

5. A. W. Jones, The lattice isomorphisms of certain finite groups, Duke Math. J. 12 (1945), 541-560.

6. M. Suzuki, Structure of a group and the structure of its lattice of subgroups, Springer-Verlag, Berlin, 1956.

7. P. Yff, On line complexes in a projective four-space, Ph.D. thesis, Univ. of Illinois, Urbana, Ill., 1957.

OHo University 